\title{
PAPER
}

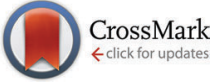

Cite this: Phys. Chem. Chem. Phys., 2016, 18, 15375

Received 29th January 2016,

Accepted 6th May 2016

DOI: $10.1039 / \mathrm{c} 6 \mathrm{cp} 00659 \mathrm{k}$

www.rsc.org/pccp

\section{Phase transitions in hydrophobe/phospholipid mixtures: hints at connections between pheromones and anaesthetic activity $\dagger$}

\author{
Silvia Borsacchi, ${ }^{a}$ Marco Geppi, ${ }^{b}$ Sara Macchi, ${ }^{b}$ Barry W. Ninham, ${ }^{c d}$ Emiliano Fratini, ${ }^{c}$ \\ Moira Ambrosi, ${ }^{c}$ Piero Baglioni ${ }^{c e}$ and Pierandrea Lo Nostro*ce

\begin{abstract}
The phase behavior of a mixture of a typical insect pheromone (olean) and a phospholipid (DOPC)/water dispersion is extensively explored through SAXS, NMR and DSC experiments. The results mimic those obtained with anaesthetics in phospholipid/water systems. They also mimic the behavior and microstructure of ternary mixtures of a membrane mimetic, bilayer-forming double chained surfactants, oils and water. Taken together with recent models for conduction of the nervous impulse, all hint at lipid involvement and the underlying unity in mechanisms of pheromone, anaesthetic and hydrophobic drugs, where a local phase change in the lipid membrane architecture may be at least partly involved in the transmission of the signal.
\end{abstract}

\section{Introduction}

Light detection by fireflies, the activity of chemical pheromones, general and local anaesthetics, cationic surfactants as immunosuppressants, cationic polymers as poisons and muscle relaxants, and other drugs, all involve interactions with the membrane of the nerve axon. The phase changes induced in the lipid membrane are all reversible. At least partly, the mechanisms must be physical, not biochemical alone. ${ }^{1}$

The implications are that the induced microstructure provides a unified mechanism that interplays with several biophysical processes such as nerve conduction, anaesthetic and pheromone activities.

The outline of this paper is as follows:

We briefly recall in the Introduction the implication of phase changes in anaesthetics and pheromone activity and we shortly present the experimental approach of this work. We then report our results on the uptake of a highly hydrophobic molecule into a biomimetic lipid bilayer and the resulting phase changes that

\footnotetext{
${ }^{a}$ Istituto di Chimica dei Composti OrganoMetallici (ICCOM) del CNR, 56124 Pisa, Italy

${ }^{b}$ Department of Chemistry and Industrial Chemistry, University of Pisa, 56124 Pisa, Italy

${ }^{c}$ Department of Chemistry \& CSGI, University of Florence, 50019 Sesto Fiorentino (Firenze), Italy. E-mail: PLN@csgi.unifi.it; Fax: +39 055457 3036; Tel: +39055 4573010

${ }^{d}$ Department of Applied Mathematics, Research School of Physical Sciences and Engineering, Australian National University, Canberra, ACT 0200, Australia

${ }^{e}$ Enzo Ferroni Foundation, 50019 Sesto Fiorentino (Firenze), Italy

$\dagger$ Electronic supplementary information (ESI) available. See DOI: 10.1039/c6cp00659k
}

occur. In the end, we discuss the experimental results in light of a combined mechanism, which at least partly involves physical chemistry and not biochemistry alone.

\subsection{Anaesthetics}

If the lipid phase transition model captures some essentials of the mechanism, and specifically, involves a cubic-lamellar phase transition, then the activity of anaesthetics may be at least partly attributed to changes in the membrane structure induced by adsorption of a hydrophobe.

The mechanism by which general anaesthetics operate is still unknown and controversial. ${ }^{2,3}$

Neither the Meyer-Overton rule (or lipid theory), nor the protein theory can explain satisfactorily all the experimental evidence. ${ }^{3}$ The former is based on the general hydrophobic nature of anaesthetics that results in their great solubility in the hydrocarbon chain pocket. The latter is based on the evidence that anaesthetics bind specifically with proteins such as serum albumin and adenylate kinase, and activate receptors or ion channels. The interaction of different local and general anaesthetics with vesicles ${ }^{4-6}$ and planar bilayers ${ }^{7,8}$ supported on solid substrates has been approached in previous contributions. The interested reader can refer to some relevant reviews on the subject. ${ }^{9-12}$ Here we just recall that (i) the effect of anaesthetics seems to be related to the hydration of biomembranes, ${ }^{13,14}$ (ii) effective anaesthetics $\left(\right.$ e.g. $\left.\mathrm{CHCl}_{3}\right)$ need to be at least slightly polar to reside close to the interface of the bilayer, ${ }^{3,13,15-32}$ (iii) the active molecule directly interacts with the membrane lipids and thus modifies the fluidity, the curvature and the phase structure of the bilayer which in turn alters the conformation and 
functional performances of ion channels, receptors and more in general of transmembrane proteins, ${ }^{3,16-22}$ and (iv) finally according to a more recent view, certain membrane proteins are clustered in cholesterol-rich "lipid rafts", i.e. transient micro-phase separated domains in a liquid ordered phase $\left(\mathrm{L}_{\mathrm{o}}\right)$, with characteristics intermediate between those of a gel and of a liquid-crystalline phase, in coexistence with a fluid-like liquid disordered phase $\left(\mathrm{L}_{\mathrm{d}}\right) \cdot{ }^{23,24}$ In passing we recall that the effect of small hydrophobic chemicals such as chloroform or diethyl ether on phospholipid model membranes has been the object of a great number of studies as for example in ref. 22 and 26.

Probably the origin of anaesthetic activity is manifold and there is no single switch to induce anesthesia. As clinical experiments suggest, multiple neurotransmission factors respond to anaesthetics with high cooperativeness, and presumably the lipid rearrangement is one of them. ${ }^{28}$

\subsection{Pheromones}

Pheromones are molecules that are produced by one individual and act as ectohormones to hijack behavior when detected by another member of the same species. ${ }^{33}$ Several pheromones, particularly those produced by insects, are lipid molecules such as ketones, aldehydes and fatty acids, ${ }^{34}$ which are more or less volatile. ${ }^{35}$ Usually volatile pheromones are detected by olfactory receptors, while low- or non-volatile pheromones are detected via contact chemosensory receptors distributed across the body. ${ }^{35}$ In particular queen pheromones that signal the presence of a fertile queen and induce daughter workers to remain sterile as in bees, termites, ants, etc., contain both volatile and non-volatile components. ${ }^{36}$ The way pheromones work is the object of several studies. Recently it was found that free fatty acids act as ligands for gypsy moth's pheromone and control their adsorption on hydrophobic surfaces. ${ }^{37}$

The work in this paper explores the phase behavior of hydrophobic pheromone/phospholipid mixtures in water dispersions. We note that there is no difference in the chemistry of pheromone or anaesthetic molecules. We deduce that there is nothing magic with either in their influence in modifying the lipid membrane nanostructure. That observation sits well with the known fact that pheromones in excess do behave as anaesthetics. ${ }^{38}$

On these premises we decided to explore membrane changes induced by an anaesthetic-like pheromone. The molecule chosen was 1,7-dioxaspiro[5.5] undecane or "olean" (Fig. 1), the major component of the sex pheromone of olive fly (Bactrocera oleae Gmelin). According to some preliminary tests, olean is a potent killer of other insect species (e.g. mosquitoes) at relatively high

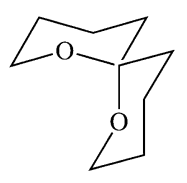

(R)

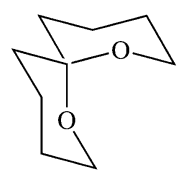

(S)
Fig. 1 Chemical structure of 1,7-dioxaspiro[5.5] undecane ("olean"). concentrations (data to be published). In any event, we have reasonable expectations that any pheromone induced phase behavior may well mimic that of anaesthetic molecules and provide hints at the mechanism of both.

\subsection{Experimental approach}

In order to investigate in detail the phase changes induced in a biomimetic lipid bilayer by the presence of a pheromone, we performed small angle X-ray scattering (SAXS), differential scanning calorimetry (DSC) and multinuclear magnetic resonance spectroscopy (NMR) experiments on a series of samples containing an aqueous dispersion of dioleoylphosphatidylcholine (DOPC), and increasing amounts of olean. Moreover, dispersions of DOPC in deuterated water, with different concentrations of olean, were prepared and studied through ${ }^{2} \mathrm{H}-\mathrm{NMR}$. In addition, aqueous dispersions of DOPC containing deuterated cyclohexane $\left(\mathrm{d}_{12}\right)$ were prepared and investigated. Since the deuterated form of olean is not commercially available, and because of its cyclic structure and similar polarity, cyclohexane was chosen to mimic the pheromone molecule.

\section{Experimental section}

\subsection{Chemicals}

1,7-Dioxaspiro[5.5] undecane or “olean” (Fig. 1) was purchased from Alfa Aesar (Heysham, United Kingdom; purity > 98\%) and used without further purification.

Dioleoylphosphatidylcholine (DOPC) was supplied by Avanti Polar Lipids (Birmingham, Alabama) and used without further purification. Cyclohexane- $\mathrm{d}_{12}$ and deuterium oxide were supplied by Sigma-Aldrich (Milan, Italy) and used as received.

Sample preparation. A $60 \% \mathrm{w} / \mathrm{w}$ stock dispersion of DOPC/ $\mathrm{H}_{2} \mathrm{O}$ and DOPC/ $\mathrm{D}_{2} \mathrm{O}$ (molar ratio $1: 30$ ) was prepared. At this concentration and at $25{ }^{\circ} \mathrm{C}$ the system forms a lamellar phase. ${ }^{39}$ The samples were annealed through repeated heating-cooling cycles, then centrifuged and stirred. The same treatment was applied to the samples containing the hydrophobe, either the pheromone or cyclohexane- $\mathrm{d}_{12}$, with a hydrophobe/DOPC molar ratio ranging between $0: 1$ and $10: 1$. All the samples were left to equilibrate for a week in a refrigerator before performing the experiments. All samples at all concentrations were - at least macroscopically - homogeneous. No phase separation was ever detected after the sample preparation.

\subsection{NMR}

${ }^{31} \mathrm{P},{ }^{2} \mathrm{H},{ }^{13} \mathrm{C}$ and ${ }^{1} \mathrm{H}$-NMR spectra were acquired on a Varian Infinity Plus 400 spectrometer, working at a Larmor frequency of 161.94, 61.41, 100.58 and $400.03 \mathrm{MHz}$ for ${ }^{31} \mathrm{P},{ }^{2} \mathrm{H},{ }^{13} \mathrm{C}$ and ${ }^{1} \mathrm{H}$, respectively, equipped with a $5 \mathrm{~mm}$ goniometric probe.

The $90^{\circ}$ pulse duration for ${ }^{31} \mathrm{P},{ }^{2} \mathrm{H},{ }^{13} \mathrm{C}$ and ${ }^{1} \mathrm{H}$ NMR was 3.0, $3.5,4.0$ and $4.4 \mu \mathrm{s}$, respectively. All the spectra were acquired under static conditions. ${ }^{31} \mathrm{P}$ spectra were acquired with ${ }^{31} \mathrm{P}$ direct excitation with high power decoupling from ${ }^{1} \mathrm{H}$, with a recycle delay of $5 \mathrm{~s}$, and accumulating 2048 transients. ${ }^{2} \mathrm{H}$ spectra were acquired with the quadrupolar echo pulse 


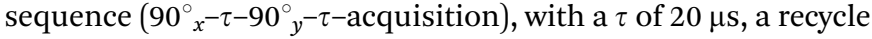
delay of $2.5 \mathrm{~s}$, and accumulating 32768 transients. ${ }^{13} \mathrm{C}$ direct excitation spectra were acquired using a DEPTH pulse sequence for eliminating the probe background signal, ${ }^{40}$ with high-power decoupling from protons, using a recycle delay of $3 \mathrm{~s}$, and accumulating 4096 transients. ${ }^{1} \mathrm{H} T_{1}$ were measured with the saturation recovery sequence, with delays varying from 0.01 to $30 \mathrm{~s} .{ }^{2} \mathrm{H} T_{1}$ were measured with the inversion recovery sequence with delays varying from 0.1 to $2 \mathrm{~s} .{ }^{31} \mathrm{P}$ spectral simulations were performed using WSOLIDS software developed in the group of Roderick Wasylishen. ${ }^{41}{ }^{31} \mathrm{P},{ }^{2} \mathrm{H}$ and ${ }^{13} \mathrm{C}$ chemical shifts were referenced to phosphoric acid ( $85 \mathrm{wt} \%, 0 \mathrm{ppm}$ ), $\mathrm{D}_{2} \mathrm{O}(0 \mathrm{ppm})$ and dodecane used as secondary references, respectively, and to TMS employed as the primary reference. All experiments were carried out at $25{ }^{\circ} \mathrm{C}$.

\subsection{SAXS}

Small angle X-ray scattering (SAXS) measurements were carried out using an HECUS SWAX-camera (Kratky) equipped with a position-sensitive detector (OED 50M) containing 1024 channels of width $54 \mu \mathrm{m}$. $\mathrm{Cu} \mathrm{K} \alpha$ radiation of wavelength, $\lambda=1.542 \AA$, was provided by a Seifert ID-3003 X-ray generator (sealed-tube type), operating at a maximum power of $2 \mathrm{~kW}$. A $10 \mu \mathrm{m}$ thick nickel filter was used to eliminate the $\mathrm{Cu} \mathrm{K} \beta$ radiation. The sample-to-detector distance was $273 \mathrm{~mm}$. The volume between the sample and the detector was kept under vacuum during the measurements to minimize scattering from air. The Kratky camera was calibrated in the small angle region using silver behenate $(d=58.38 \AA)^{42}$ Scattering curves were obtained in the $Q$-range between 0.01 and $0.54 \AA^{-1}, Q$ being the scattering vector $4 \pi(\sin \theta) / \lambda$, and $2 \theta$ the scattering angle. Paste-like samples were filled into a $1 \mathrm{~mm}$ demountable cell having kapton-film as windows. The temperature was controlled by a Peltier element, with an accuracy of $\pm 0.1^{\circ} \mathrm{C}$. All scattering curves were corrected for the empty cell contribution considering the relative transmission factor. SAXS curves were iteratively de-smeared using the procedure reported by Lake. ${ }^{43}$

\subsection{DSC}

Differential scanning calorimetry (DSC) runs were performed on Q1000 (TA Instruments) apparatus using aluminum hermetic pans. For each phase transition the peak temperature and the corresponding enthalpy change $(\Delta H)$ were evaluated. The samples

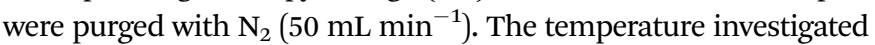
ranged between $-60{ }^{\circ} \mathrm{C}$ and $40{ }^{\circ} \mathrm{C}$, at a heating rate of $2{ }^{\circ} \mathrm{C} \mathrm{min}{ }^{-1}$. A slower scan rate did not modify the thermogram profile.

\section{Results and discussion}

\subsection{SAXS}

SAXS measurements (Fig. 2) confirm that a $60 \% \mathrm{w} / \mathrm{w}$ dispersion of DOPC in water, our biomimetic lipid bilayer, consists of a lamellar phase $\left(\mathrm{L}_{\alpha}\right)$ with a spacing $d=51.50 \AA \AA^{39}$

When the pheromone is uploaded at a 1:1 molar ratio the SAXS pattern still indicates the presence of a lamellar phase, but with an increment in the $d$ spacing up to $53.07 \AA$ (squares in Fig. 2).

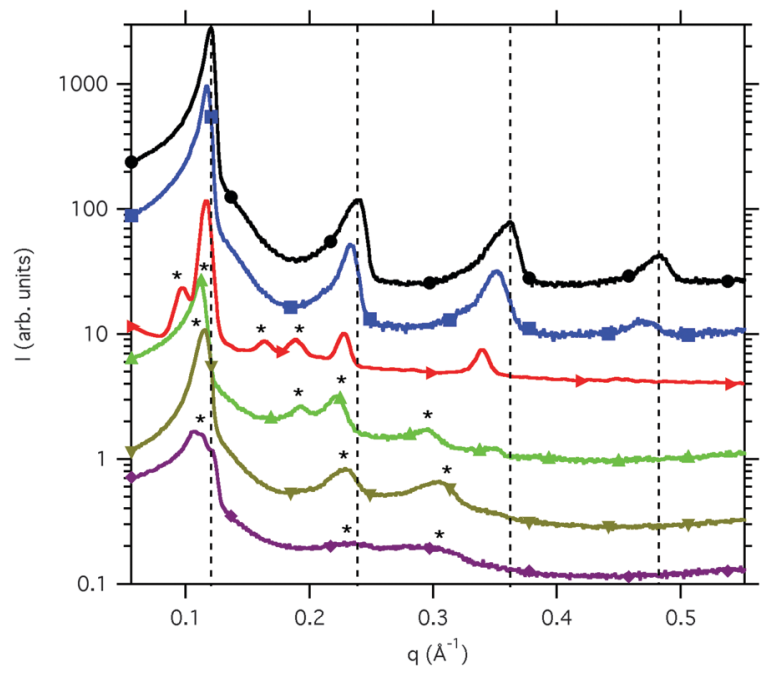

Fig. 2 SAXS profiles of a pure $60 \% \mathrm{w} / \mathrm{w}$ DOPC aqueous dispersion ( $)$, at

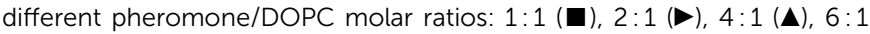
$(\mathbf{\nabla})$, and $10: 1(\bullet)$. The asterisks denote the peaks due to the inverse hexagonal phase.

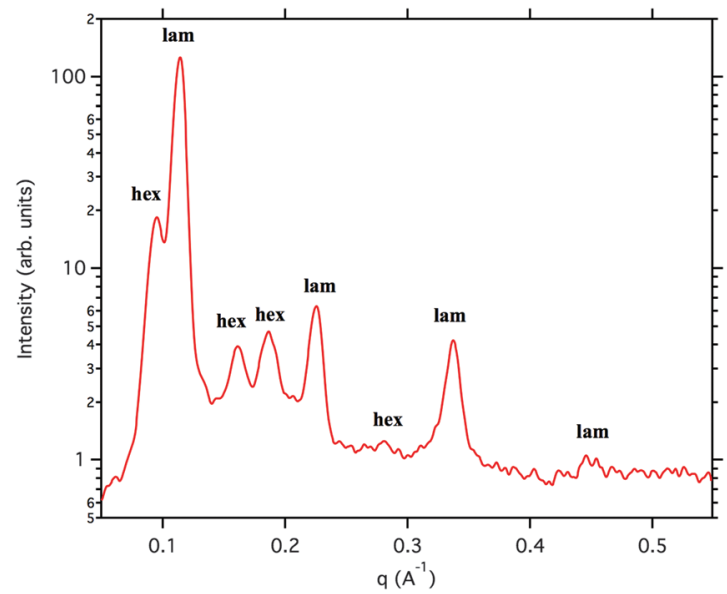

Fig. 3 SAXS profile for $60 \% \mathrm{w} / \mathrm{w}$ DOPC aqueous dispersion containing a 2:1 pheromone/DOPC molar ratio. The peaks typical for a lamellar (lam) and an inverse hexagonal (hex) phase are indicated.

Fig. 3 shows the SAXS profile for a pheromone/DOPC molar ratio of $2: 1$, suggesting the coexistence of lamellar $\left(\mathrm{L}_{\alpha}\right)$ and inverse hexagonal $\left(\mathrm{H}_{\mathrm{II}}\right)$ phases. This result will be confirmed by ${ }^{31} \mathrm{P}$ NMR experiments (see below).

For a pheromone/DOPC molar ratio of $4: 1$ the SAXS profile indicates the presence of an inverse hexagonal phase with a spacing of $64.10 \AA$ (up-pointing triangles in Fig. 2).

A further increment in the pheromone concentration induces a reduction in the extension of the ordered domain and in the $d$ spacing, down to $62.12 \AA$ for a molar ratio of $6: 1$ and to $60.46 \AA$ for a molar ratio of $10: 1$. The broadening of the peaks reflects a significant loss in the long range structure.

Table 1 summarizes the DOPC/water phases observed for the different pheromone/DOPC molar ratios $(c)$ and the corresponding spacings $(d)$ obtained from SAXS measurements. 
Table $1 d$ spacings (in $\AA$ ) and phase description for the investigated DOPC/water/pheromone samples at different pheromone/DOPC molar ratios (c)

\begin{tabular}{lll}
\hline$c$ & $d(\AA)$ & Phase \\
\hline $0: 1$ & 51.5 & $\mathrm{~L}_{\alpha}$ \\
$1: 1$ & 53.1 & $\mathrm{~L}_{\alpha}$ \\
$2: 1$ & 55.3 & $\mathrm{~L}_{\alpha}$ \\
$2: 1$ & 66.5 & $\mathrm{H}_{\text {II }}$ \\
$4: 1$ & 64.1 & $\mathrm{H}_{\text {II }}$ \\
$6: 1$ & 62.1 & $\mathrm{H}_{\text {II }}$ \\
$10: 1$ & 60.5 & $\mathrm{H}_{\text {II }}$
\end{tabular}

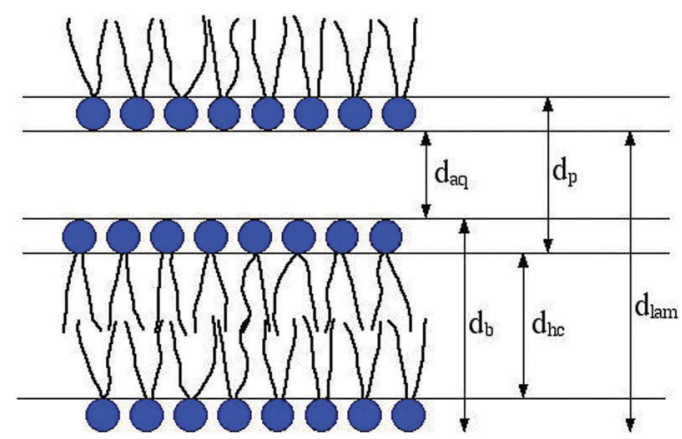

Fig. 4 Structure of a lamellar $\left(\mathrm{L}_{\alpha}\right)$ phase. $d_{\text {lam }}$ is the experimental spacing directly obtained from SAXS measurements (see Table 2). $d_{\mathrm{hc}}$ is twice the length of the hydrocarbon chains, $d_{\mathrm{b}}$ is twice the length of the entire phospholipid, $d_{\mathrm{aq}}$ is the thickness of the water pool, and $d_{\mathrm{p}}$ is the thickness of the polar region (including the two facing layers of polar heads). $A_{p}$ is the polar head cross-section. $d_{\mathrm{hc}}, d_{\mathrm{b}}, d_{\mathrm{aq}}, d_{\mathrm{p}}$ and $A_{\mathrm{p}}$ can be obtained from $d_{\text {lam }}$ through eqn (1)-(5). Adapted with permission from ref. 44. Copyright 1994, Elsevier Ltd.

Fig. 4 and 5 show the structure of $\mathrm{L}_{\alpha}$ and $\mathrm{H}_{\mathrm{II}}$ phases, respectively, and the structural parameters that can be calculated from the experimental spacings $d$, indicated as $d_{\text {lam }}$ and

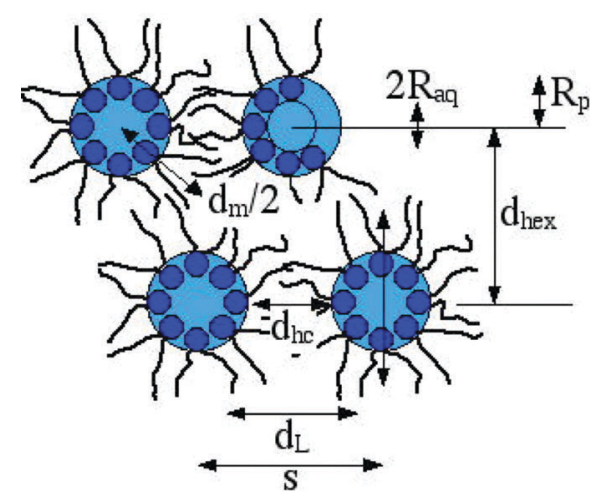

Fig. 5 Structure of an inverse hexagonal phase $\left(\mathrm{H}_{\| 1}\right) . d_{\text {hex }}$ is the experimental spacing directly obtained from SAXS measurements (see Table 3). $R_{\mathrm{aq}}$ is the radius of the water inner pool, $R_{\mathrm{p}}$ is the radius of the hydrophilic compartment, including the thickness of the polar head, $d_{m}$ is the distance between the centers of two diagonal cylinders, $s$ is the center-to-center distance between two adjacent cylinders, and $d_{\mathrm{L}}$ is given by $d_{\mathrm{hc}}$ and twice the thickness of the polar head. $R_{\mathrm{aq}}, R_{\mathrm{p}}, d_{\mathrm{m}}, s$ and $d_{\mathrm{L}}$ can be obtained from $d_{\text {hex }}$ through eqn (6)-(9). Adapted with permission from ref. 44. Copyright 1994, Elsevier Ltd. $d_{\text {hex }}$ for the $\mathrm{L}_{\alpha}$ and $\mathrm{H}_{\mathrm{II}}$ phases, respectively, according to eqn (1)-(9). ${ }^{44}$

$$
\begin{gathered}
d_{\mathrm{b}}=\frac{v_{\mathrm{l}}+c v_{\mathrm{phe}}}{v_{\mathrm{aq}}+v_{\mathrm{l}}+c v_{\mathrm{phe}}} d_{\mathrm{lam}}=\phi d_{\mathrm{lam}} \\
A_{\mathrm{p}}=\frac{2\left(v_{\mathrm{l}}+c v_{\mathrm{phe}}\right)}{d_{\mathrm{b}}} \\
d_{\mathrm{hc}}=\frac{2\left(v_{\mathrm{hc}}+c v_{\mathrm{phe}}\right)}{A_{\mathrm{p}}} \\
d_{\mathrm{aq}}=d_{\mathrm{lam}}-d_{\mathrm{b}} \\
d_{\mathrm{p}}=\frac{2\left(v_{\mathrm{aq}}+v_{\mathrm{p}}\right)}{A_{\mathrm{p}}} \\
s=\frac{2 d_{\mathrm{hex}}}{\sqrt{3}} \\
d_{\mathrm{m}}=2\left(\frac{s}{\left.\sqrt{3}-R_{\mathrm{aq}}\right)}\right. \\
d_{\mathrm{L}}=s-2 R_{\mathrm{aq}} \\
\pi \sqrt{3} d_{\mathrm{hex}}^{2}(1-\phi) \\
\end{gathered}
$$

where $v_{1}$ is the volume of one phospholipid molecule, $\phi$ is the volume fraction of the phospholipid and the pheromone, $v_{\mathrm{aq}}$ is the volume of water per phospholipid molecule, $v_{\text {hc }}$ is the volume of the hydrocarbon chains, $v_{\text {phe }}$ is the volume of the pheromone, $c$ is the pheromone/DOPC molar ratio and $v_{\mathrm{p}}$ is the volume of the polar part. ${ }^{44}$ Since the pheromone molecules are completely soluble in an apolar solvent such as $n$-decane, we assume that they are located in the hydrophobic portion of the bilayer and the inverse hexagonal phase. Moreover, since the olean molecule is hindered and stiff, we assume that once in the lamellar or inverse hexagonal phase, it will retain its molecular volume as in the pure liquid state. For the calculations, the molecular volume of water is taken to be $30 \AA^{3}$ at $20{ }^{\circ} \mathrm{C}$ and the number of hydration molecules around the DOPC polar head is 9. ${ }^{39}$ The volume of a single DOPC molecule $\left(v_{1}\right)$ is $1290 \AA^{3}$, the volume occupied by the polar head $\left(v_{\mathrm{p}}\right)$ is $374 \AA^{3}$, while that occupied by the hydrophobic chains $\left(v_{\mathrm{hc}}\right)$ is $916 \AA^{3} .^{44,45}$ The volume of a pheromone molecule in the liquid state $\left(v_{\text {phe }}\right)$ was calculated from its density and is $254 \AA^{3}$.

Tables 2 and 3 list the structural parameters and the volume fractions for the samples investigated.

The data obtained for pure DOPC/water agree with those reported in the literature. ${ }^{39,46}$

The uptake of the pheromone molecules brings about an increment of $4 \AA$ in $d_{\text {hc }}$ spacing as a consequence of the thicker hydrophobic portion of the lipid bilayer in the lamellar phase. In fact $d_{\mathrm{b}}$ and $d_{\mathrm{hc}}$ increase, while $d_{\mathrm{aq}}$ and $d_{\mathrm{p}}$ decrease.

In the case of the inverse hexagonal phase (Fig. 5 and 6) the further addition of pheromone to the sample with a 
Table 2 Structural parameters of the lamellar phase (see Fig. 4) at two different pheromone/DOPC molar ratios (c) calculated according to eqn (1)-(5)

\begin{tabular}{llllllll}
\hline$c$ & $\phi$ & $d_{\text {lam }}(\AA)$ & $d_{\mathrm{b}}(\AA)$ & $A_{\mathrm{p}}\left(\AA^{2}\right)$ & $d_{\mathrm{aq}}(\AA)$ & $d_{\mathrm{p}}(\AA)$ & $d_{\mathrm{hc}}(\AA)$ \\
\hline $0: 1$ & 0.8269 & 51.5 & 42.6 & 60.6 & 8.9 & 21.3 & 30.2 \\
$1: 1$ & 0.8512 & 53.1 & 45.2 & 68.4 & 7.9 & 18.8 & 34.2
\end{tabular}

Table 3 Structural parameters for the inverse hexagonal phase (see Fig. 5) at different pheromone/DOPC molar ratios (c) calculated according to eqn (6)-(9)

\begin{tabular}{llllcll}
\hline$c$ & $\phi$ & $d_{\text {hex }}(\AA)$ & $s(\AA)$ & $R_{\text {aq }}(\AA)$ & $d_{\mathrm{L}}(\AA)$ & $d_{\mathrm{m}}(\AA)$ \\
\hline $4: 1$ & 0.8952 & 64.1 & 74.0 & 12.6 & 48.8 & 60.3 \\
$6: 1$ & 0.9125 & 62.1 & 71.7 & 11.1 & 49.5 & 60.6 \\
$10: 1$ & 0.9341 & 60.5 & 69.9 & 9.4 & 51.1 & 61.9
\end{tabular}

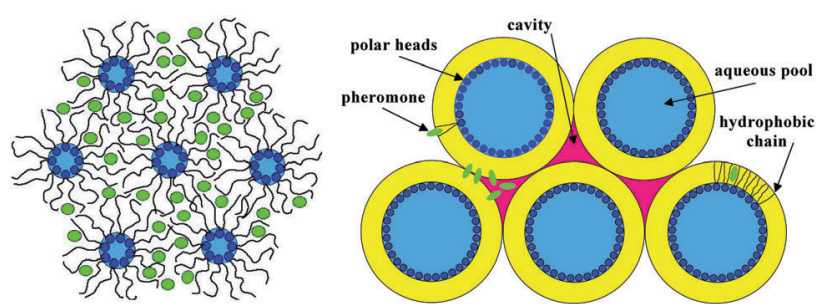

Fig. 6 Inverse hexagonal array in the aqueous dispersion of DOPC after the uptake of the pheromone molecules (in green). The dark blue spots represent the polar heads of the phospholipid, while the light blue regions indicate the inner water pool, the yellow layers are the hydrophobic portion of the nanostructure, and the red area is the inter-cylinder region occupied by the pheromone molecules.

pheromone/DOPC molar ratio (c) of $4: 1$ reduces the thickness $d_{\text {hex }}$ and the distance between the centres, $s$, of the cylinders of about $4 \AA$ (see Table 3).

This change produces a more compact structure, with a reduction of the water pool size from 12.6 to $9.4 \AA$ A. On the other hand, the distance $d_{\mathrm{m}}$ increases from 60.3 to $61.8 \AA$. These results suggest that the pheromone molecules occupy the cavity between the cylinders, as shown in Fig. 6, with significant stabilization of the structure. ${ }^{47}$

For $c$ greater than 10:1 the amount of pheromone is so large that it cannot be dispersed into the cavities, and the sample loses its long range structure, as suggested by the broad peak in the SAXS pattern (diamonds in Fig. 2).

\subsection{DSC}

The thermograms of the investigated DOPC/water/pheromone samples show the presence of two peaks during the cooling and two peaks during the heating scan. In order to avoid problems related to overcooling effects, we have considered only the heating peaks (see Fig. S1 in the ESI $\dagger$ ).

In the case of a $60 \%$ aqueous dispersion of DOPC the liquid crystal-lamellar phase transition $\left(\mathrm{L}_{\mathrm{c}}-\mathrm{L}_{\alpha}\right)$ is found at $-16.8{ }^{\circ} \mathrm{C}$, with a corresponding enthalpy change of about $35.0 \mathrm{~kJ} \mathrm{~mol}_{\mathrm{DOPC}}{ }^{-1}$ (Fig. 7). These values agree with those reported in the literature for

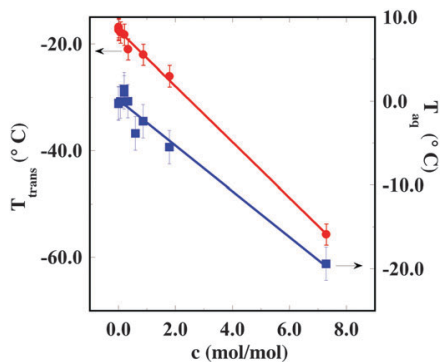

Fig. $7 \quad L_{c}-L_{\alpha}$ phase transition temperature ( $T_{\text {trans, }}$ red line) and water melting temperature ( $T_{\mathrm{aq}}$, blue line) as a function of the pheromone/DOPC molar ratio (c). The error bars represent the standard deviation.

a water/DOPC molar ratio of $30 .^{39,48}$ The melting of water in the pure DOPC aqueous dispersion occurs at $-0.2{ }^{\circ} \mathrm{C}$ (Fig. 7).

As reported by Ulrich the sub-zero melting of the phospholipid's acyl chains occurs in the presence of frozen ice, instead of liquid water. ${ }^{39}$ This is the reason why the thermal behavior of unsaturated lipids differs from that of saturated lipids. In turn, when ice melts around $0{ }^{\circ} \mathrm{C}$ the unsaturated hydrophobic tails are in a liquid state ( $\mathrm{L}_{\alpha}$ phase). This occurrence, together with the thermally induced fluctuations and undulations in the bilayer, brings about an increment in the head group cross-section that results in a lowering of the chemical potential of the hydrating water molecules that reside near the bilayer interface. ${ }^{26,49-51}$ Hence, the water melting point decreases and the polar heads undergo relatively small conformational adjustment. ${ }^{39,52}$ Furthermore, the addition of the hydrophobic pheromone to the DOPC dispersion increases the disorder in the hydrophobic layer and therefore decreases the $\mathrm{L}_{\mathrm{c}} \rightarrow \mathrm{L}_{\alpha}$ phase transition temperature. Further additions of pheromone induce the formation of the $\mathrm{H}_{\text {II }}$ phase and lower the $\mathrm{L}_{\alpha} \rightarrow \mathrm{H}_{\mathrm{II}}$ transition temperature. ${ }^{53}$

The water melting temperature remains almost constant (see the blue line in Fig. 7) up to the $1: 1$ pheromone/DOPC molar ratio, and then decreases down to $-19.4{ }^{\circ} \mathrm{C}$. We recall that water pore-confined into the rod-like micelles of the $\mathrm{H}_{\mathrm{II}}$ phase freezes below $0{ }^{\circ} \mathrm{C} .{ }^{54}$

The enthalpy change $\left(\Delta H_{\text {trans }}\right)$ associated with the $\mathrm{L}_{\mathrm{c}}-\mathrm{L}_{\alpha}$ transition does not vary significantly until a pheromone/DOPC molar ratio of $8: 1$ is reached, where a cubic phase is formed, as indicated by the NMR measurements (see below).

\subsection{NMR}

NMR experiments were performed on DOPC $/ \mathrm{D}_{2} \mathrm{O} /$ pheromone and DOPC/ $\mathrm{H}_{2} \mathrm{O} /$ cyclohexane- $\mathrm{d}_{12}$ samples, as a function of the pheromone and the deuterated cyclohexane concentration.

3.3.1 ${ }^{31} \mathrm{P}$ NMR. ${ }^{31} \mathrm{P}$ static spectra allow a straightforward determination of the chemical shift tensor of ${ }^{31} \mathrm{P}$ nuclei that are particularly effective in identifying the phases formed in phospholipid/water dispersions. ${ }^{55,56}$

The spectra recorded for the pure DOPC $/ \mathrm{D}_{2} \mathrm{O}$ dispersion and after the uptake of different amounts of pheromone are reported in Fig. 8 along with the corresponding spectral simulations: the spectral parameters obtained from the simulations are reported in Table 4. 

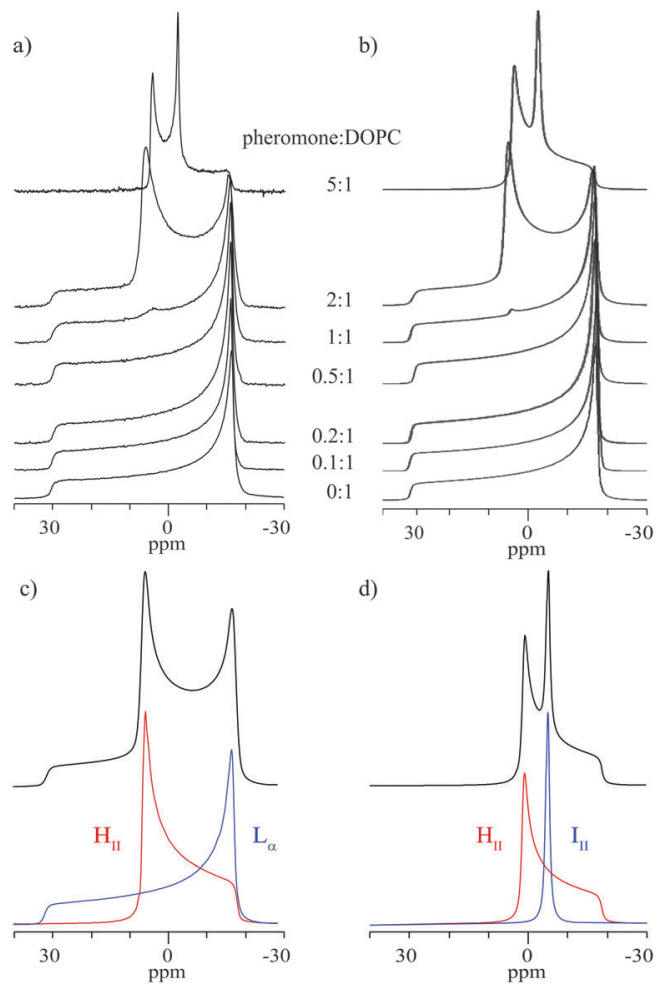

Fig. 8 Upper part: Experimental (left) and simulated (right) ${ }^{31} \mathrm{P}$ NMR spectra of $60 \% \mathrm{w} / \mathrm{w}$ DOPC $/ \mathrm{D}_{2} \mathrm{O}$ dispersion with different concentrations of pheromone. Lower part: Simulated ${ }^{31} \mathrm{P} N \mathrm{NR}$ spectra of two DOPC/D $\mathrm{D}_{2} \mathrm{O} /$ pheromone samples highlighting the spectral contributions of $L_{\alpha}, H_{\|}$and $I_{\|}$ phases.

The lineshape of the spectrum for the DOPC $/ \mathrm{D}_{2} \mathrm{O}$ system clearly indicates the presence of a single $\mathrm{L}_{\alpha}$ phase ${ }^{55,56}$ confirming the SAXS results. By adding increasing amounts of pheromone the ${ }^{31} \mathrm{P}$ spectrum remains substantially unchanged up to a pheromone/DOPC molar ratio of $0.5: 1$. For the $1: 1$ ratio a very small amount of a second phase is revealed by the small signal at about $10 \mathrm{ppm}$. This signal becomes more evident at higher pheromone amounts. The spectral parameters obtained by simulation clearly indicate that this second phase is an inverse hexagonal phase $\mathrm{H}_{\mathrm{II}}$. At a much higher pheromone content ( $5: 1$ ratio) the $\mathrm{H}_{\text {II }}$ phase coexists with an isotropic inverse cubic $\mathrm{I}_{\text {II }}$ phase, which gives rise to the narrow isotropic signal superimposed onto that of the $\mathrm{H}_{\mathrm{II}}$ phase. The same experiments were performed on the DOPC/ $\mathrm{H}_{2} \mathrm{O} /$ cyclohexane- $\mathrm{d}_{12}$ system. DOPC/ $\mathrm{H}_{2} \mathrm{O}$ gave exactly the same spectrum as DOPC/ $\mathrm{D}_{2} \mathrm{O}$.

The uptake of cyclohexane- $\mathrm{d}_{12}$ had a very similar effect on the phase behavior of the whole system, with the onset of an $\mathrm{L}_{\alpha}-\mathrm{H}_{\mathrm{II}}$ transition for the sample with a 1:1 molar ratio $\left(2 \%\right.$ of $\mathrm{H}_{\mathrm{II}}$, regularly increasing at higher cyclohexane amounts), in perfect agreement with the results obtained for the DOPC/ $\mathrm{D}_{2} \mathrm{O} /$ pheromone samples (spectra not shown). The only difference between the two sets of samples is that no isotropic $\mathrm{I}_{\text {II }}$ phase is formed even at an 8:1 pheromone/DOPC molar ratio. The strong likeness of the phase behavior of these two sets of samples further supports the choice of cyclohexane as a deuterated hydrophobic model molecule for mimicking the effects produced by the pheromone.
Table 4 Phase formed, its percentage and chemical shift tensor $\delta$ parameters of the corresponding DOPC ${ }^{31} \mathrm{P}$ sites in $60 \% \mathrm{w} / \mathrm{w} \mathrm{DOPC} / \mathrm{D}_{2} \mathrm{O}$ dispersions containing different amounts of pheromone, as obtained from simulations of the experimental ${ }^{31} \mathrm{P}$ NMR spectra. The chemical shift parameters are indicated according to the Herzfeld-Berger convention: ${ }^{.7}$ $\delta_{11} \geq \delta_{22} \geq \delta_{33}$ (in ppm) are the principal values of the $\delta$ tensor, $\Omega(\mathrm{ppm})=$ $\delta_{11}-\delta_{33}, \delta_{\text {ISO }}(\mathrm{ppm})=\left(\delta_{11}+\delta_{22}+\delta_{33}\right) / 3, k=3\left(\delta_{22}-\delta_{\text {ISO }}\right) / \Omega$. The uncertainties on the values of percentages, $\delta_{\text {ISO }}$ and $\Omega$ are $\pm 1, \pm 0.1$ and \pm 0.1 , respectively

\begin{tabular}{lllllr}
\hline$c$ & Phase & \% of phase & $\Omega(\mathrm{ppm})$ & $\delta_{\text {ISO }}(\mathrm{ppm})$ & $k$ \\
\hline $0: 1$ & $\mathrm{~L}_{\alpha}$ & 100 & 46.8 & 0.1 & -1 \\
$0.1: 1$ & $\mathrm{~L}_{\alpha}$ & 100 & 46.5 & 0.2 & -1 \\
$0.2: 1$ & $\mathrm{~L}_{\alpha}$ & 100 & 46.2 & 0.1 & -1 \\
$0.5: 1$ & $\mathrm{~L}_{\alpha}$ & 100 & 46.5 & 0.2 & -1 \\
$1: 1$ & $\mathrm{~L}_{\alpha}$ & 98 & 46.4 & 0.2 & -1 \\
& $\mathrm{H}_{\text {II }}$ & 2 & 21.2 & -1.1 & 1 \\
$2: 1$ & $\mathrm{~L}_{\alpha}$ & 53 & 46.8 & 0.3 & -1 \\
& $\mathrm{H}_{\text {II }}$ & 47 & 23.1 & -0.2 & 1 \\
$5: 1$ & $\mathrm{H}_{\text {II }}$ & 79 & 20.9 & -1.4 & 1 \\
& $\mathrm{I}_{\text {II }}$ & 21 & - & -1.3 & - \\
\hline
\end{tabular}

3.3.2 ${ }^{13} \mathrm{C}$ NMR. ${ }^{13} \mathrm{C}$ static spectra were recorded in order to study the structural and dynamic properties of the phospholipid chains. The spectra of DOPC/ $\mathrm{D}_{2} \mathrm{O} /$ pheromone samples, reported in Fig. 9, show a very good resolution. They were recorded under static conditions, which means that the residual chemical shift anisotropy (CSA) of the ${ }^{13} \mathrm{C}$ nuclei is very small and therefore both the pheromone and the phospholipid chains are in quite mobile environments.

The linewidth of the phospholipid chain signals is further reduced at high pheromone content (5:1 ratio), in agreement

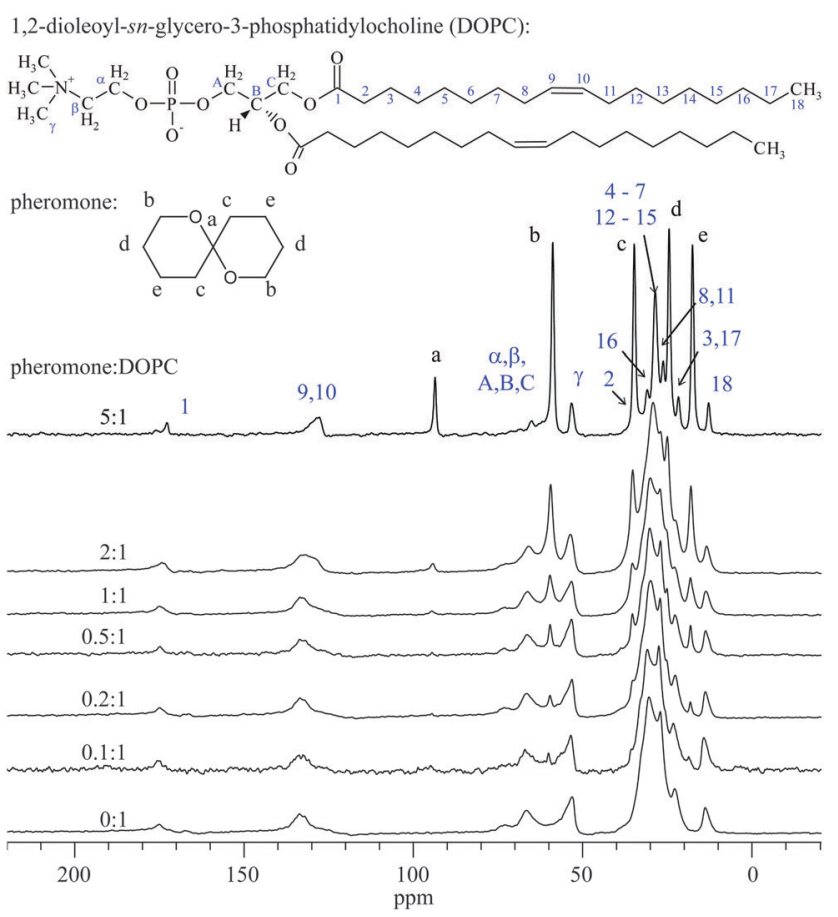

Fig. $9{ }^{13} \mathrm{C}$ spectra, recorded with high-power decoupling from ${ }^{1} \mathrm{H}$ nuclei, of $60 \% \mathrm{w} / \mathrm{w} \mathrm{DOPC} / \mathrm{D}_{2} \mathrm{O}$ dispersion with different concentrations of pheromone (reported in the figure as pheromone:DOPC molar ratios), with peak assignment. 
with the formation of the isotropic $\mathrm{I}_{\mathrm{II}}$ phase, observed from ${ }^{31} \mathrm{P}$ NMR spectra. We note that, due to the presence of the $\mathrm{C}=\mathrm{O}$ carbonyl groups and of the $\mathrm{C}=\mathrm{C}$ double bond, carbons 1,9 , and 10 (peaks at about 175 and 133 ppm) have a higher CSA, and show an inversion in the chemical shift tensor symmetry in passing from the $1: 1$ to the $2: 1$ ratio, in agreement with the $\mathrm{L}_{\alpha}-\mathrm{H}_{\mathrm{II}}$ transition.

Another interesting feature is the isotropic chemical shift of the carbon of the terminal methyl group of DOPC chains that resonates at $15.3 \mathrm{ppm}$ in the $\mathrm{DOPC} / \mathrm{D}_{2} \mathrm{O}$ dispersion: by increasing the amount of uptaken pheromone the chemical shift regularly decreases down to $14.4 \mathrm{ppm}$ in the sample with the 5:1 pheromone/DOPC molar ratio. The same effect was observed in the samples where deuterated cyclohexane replaced the pheromone (spectra not reported), suggesting that in both cases the uptaken molecules reside close to the terminal groups of the DOPC chains. This particular situation forces DOPC in the $\mathrm{L}_{\alpha}$ phase to progressively remove the interdigitation of the two aliphatic layers. This result is in good agreement with the SAXS data, which indicate an increment in the $d$ spacing of the lamellar phase with increasing pheromone concentrations. This mechanism favours the transition to the $\mathrm{H}_{\text {II }}$ phase, where the uptaken molecules moved to the hydrophobic cavities.

\subsection{3 ${ }^{2} \mathbf{H}$ NMR}

Static spectra. ${ }^{2} \mathrm{H}$ static spectra are very sensitive to the dynamic situation of deuterium nuclei and, in particular, to the rate and geometry of the motions involving the rigid fragment which the deuterium nucleus belongs to. In this study, ${ }^{2} \mathrm{H}$ spectra were recorded for the DOPC $/ \mathrm{D}_{2} \mathrm{O} /$ pheromone and DOPC $/ \mathrm{H}_{2} \mathrm{O}$ / cyclohexane- $\mathrm{d}_{12}$ mixtures with the twofold goal of obtaining dynamic information on water and hydrophobic molecules.

The spectra recorded for the DOPC/ $\mathrm{D}_{2} \mathrm{O} /$ pheromone samples are shown in Fig. 10(left).

The spectrum obtained in the absence of pheromone shows a quite "anomalous" lineshape: indeed, this is different from those previously reported in the literature for an $\mathrm{L}_{\alpha}$ phase with a similar DOPC/ $\mathrm{D}_{2} \mathrm{O}$ molar ratio $(1: 24),{ }^{58}$ arising from the superposition of an isotropic peak of extra-lamellar water and a Pake pattern due to the inter-lamellar one. However, in contrast to these findings, Zhou - mainly through ${ }^{1} \mathrm{H}$ MAS spectra - found that all the water is inter-lamellar up to a $\mathrm{DOPC} / \mathrm{H}_{2} \mathrm{O}$ ratio of $1: 40 .^{59}$

Nonetheless, our spectrum could arise from the presence of water molecules in different situations, for instance, more strongly or loosely bound to the phospholipid heads, substantially non-exchanging on the NMR time scale. The same effect on the NMR spectrum could also arise from a non-uniform distribution of inter-lamellar spacings. Another explanation is the presence of a slightly defective lamellar phase, in which some lamellae could have finite dimensions or some "holes", as observed in surfactant/water systems, ${ }^{60,61}$ or in which the bilayers could have a slight curvature, similarly to what was observed in the "ripple" phase of phospholipid/water systems. ${ }^{62}$ In any case these effects could be due to the fact that the sample did not completely reach the "equilibrium" state.

Considering all this, it must be noted that after adding a small amount of pheromone to the DOPC $/ \mathrm{D}_{2} \mathrm{O}$ system a
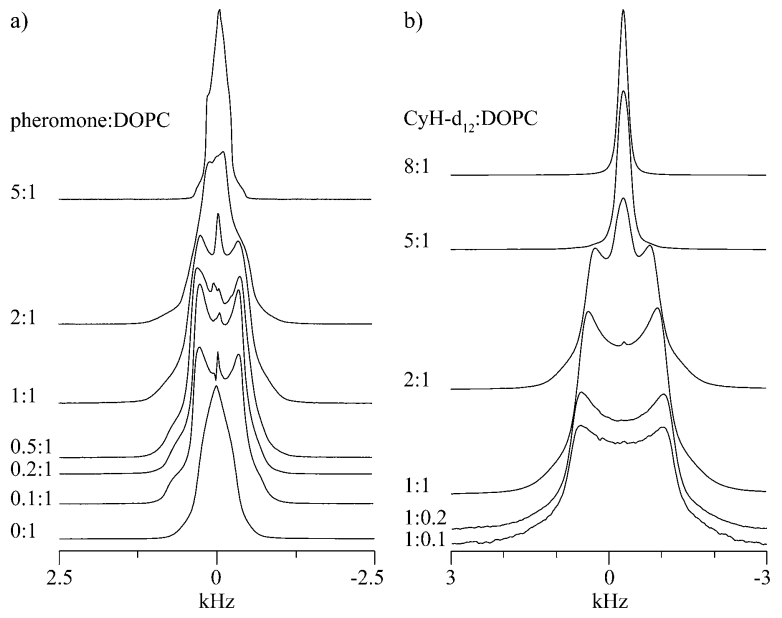

Fig. $10{ }^{2} \mathrm{H}$ NMR spectra of: (left) $60 \% \mathrm{w} / \mathrm{w}$ DOPC/ $\mathrm{D}_{2} \mathrm{O}$ dispersion with different concentrations of pheromone; (right) $60 \% \mathrm{w} / \mathrm{w}$ DOPC $/ \mathrm{H}_{2} \mathrm{O}$ dispersion with different concentrations of cyclohexane- $d_{12}$.

substantial change in the ${ }^{2} \mathrm{H}$ lineshape is observed. In particular it becomes a Pake pattern, typical for inter-lamellar water molecules in the $\mathrm{L}_{\alpha}$ phase, indicating that such phase reached a complete equilibrium. The narrow peak superimposed onto the Pake pattern is very weak and must be ascribed to condensed water vapor from moisture in the sample tubes. Therefore, in any case, no extra-lamellar water is detected, in agreement with Zhou $^{59}$ and in disagreement with Volke. ${ }^{58}$ The addition of further amounts of pheromone does not produce any relevant change in the ${ }^{2} \mathrm{H}$ lineshape up to the transition to the $\mathrm{H}_{\text {II }}$ phase occurs, as observed by ${ }^{31} \mathrm{P}$. This causes an overall reduction of the linewidth and a progressive decrease of the Pake pattern associated with the $\mathrm{L}_{\alpha}$ phase. This pattern is completely absent in the spectrum of the sample with the $5: 1$ ratio, which arises from the superposition of an anisotropic lineshape due to the $\mathrm{H}_{\text {II }}$ phase and an isotropic peak due to the $\mathrm{I}_{\mathrm{II}}$ phase, in agreement with the ${ }^{31} \mathrm{P}$ results.

The spectra recorded for the set of DOPC/ $\mathrm{H}_{2} \mathrm{O} /$ cyclohexane- $\mathrm{d}_{12}$ samples are reported in Fig. 10(right).

The lineshape obtained at the lowest concentrations arises from the partially anisotropic average of the quadrupolar interaction due to three different fast motions: rotation about the $C_{3}$ symmetry axis, tumbling of this axis with respect to the local phase director (normal to the surface of the double layer), and chair-chair interconversion. ${ }^{63}$ The experimental quadrupolar splitting corresponds to an order parameter of -0.037 , which indicates that the $C_{3}$ symmetry axis tends to have a slight preferential orientation perpendicular to the double layers. The order parameter obtained is in agreement with those reported for the terminal ends of the phospholipid chains in the double layers, ${ }^{64}$ confirming that the cyclohexane molecules are located near the methyl end groups of the hydrocarbon chains, as already inferred from the ${ }^{13} \mathrm{C}$ spectra. At higher cyclohexane concentrations an isotropic peak progressively rises, becoming the only peak when the cyclohexane: DOPC molar ratio is $5: 1$. This is in agreement with the location of cyclohexane molecules within the hydrophobic cavities in the $\mathrm{H}_{\text {II }}$ phase, where they no 

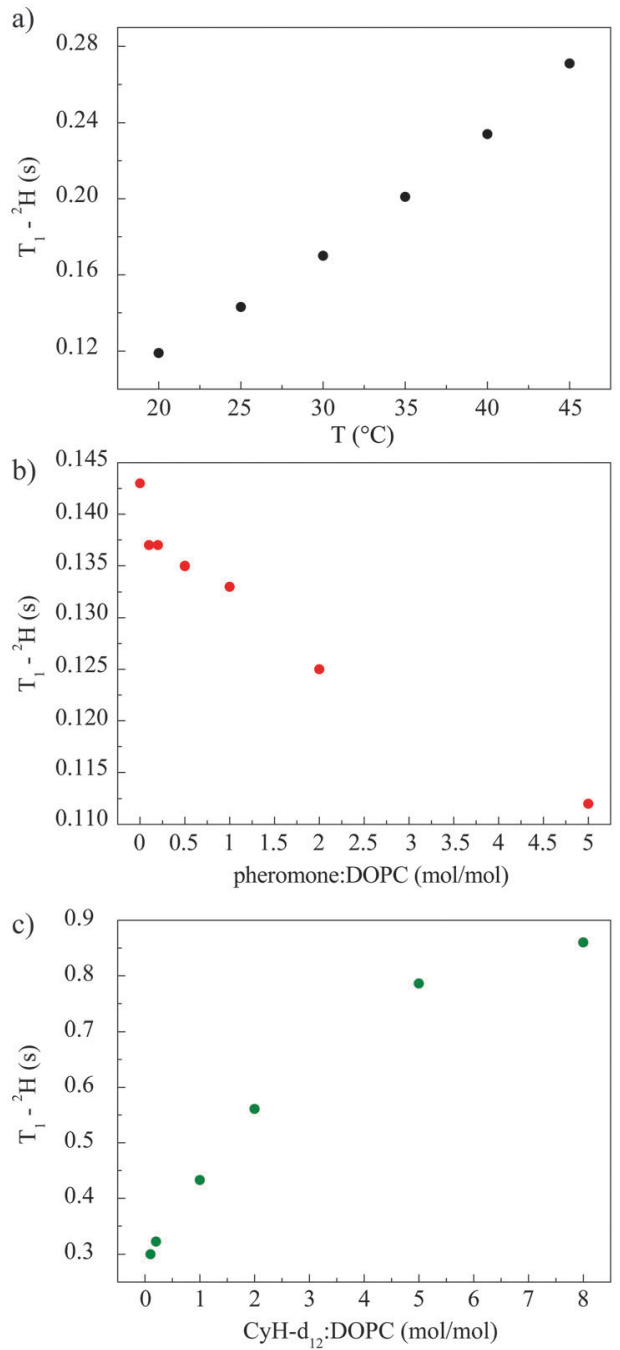

Fig. $11{ }^{2} \mathrm{H}$ spin-lattice relaxation time $T_{1}$ of: (a) deuterated water in a $60 \%$ $\mathrm{w} / \mathrm{w} \mathrm{DOPC} / \mathrm{D}_{2} \mathrm{O}$ dispersion, as a function of temperature; (b) deuterated water in a $60 \% \mathrm{w} / \mathrm{w} \mathrm{DOPC} / \mathrm{D}_{2} \mathrm{O}$ dispersion, at room temperature, as a function of the concentration of the uptaken pheromone and (c) cyclohexane- $d_{12}$ (indicated as $\mathrm{CyH}-\mathrm{d}_{12}$ ) in $60 \% \mathrm{w} / \mathrm{w} \mathrm{DOPC} / \mathrm{H}_{2} \mathrm{O}$ dispersion, at room temperature, as a function of cyclohexane- $d_{12}$ concentration.

longer have a preferential orientation and can experience isotropic reorientational motions.

3.3.4 Spin-lattice relaxation times. Additional information on the dynamic behavior of water molecules, and in particular on motions with characteristic frequencies of the order of tens of $\mathrm{MHz}$, could be obtained by measuring ${ }^{2} \mathrm{H}$ spin-lattice relaxation times $\left(T_{1}\right)^{65}$ in the DOPC/ $\mathrm{D}_{2} \mathrm{O} /$ pheromone samples. The trend of ${ }^{2} \mathrm{H} T_{1} v$ s. temperature for DOPC/ $\mathrm{D}_{2} \mathrm{O}$ is reported in Fig. 11a.

The relaxation time increases regularly with increasing temperature, clearly indicating that the motions contributing to the relaxation are in the fast motional regime (characteristic frequencies larger than ${ }^{2} \mathrm{H}$ Larmor frequency, here 61.4 MHz). Hence the decreasing trend detected for this relaxation time with increasing pheromone content (Fig. 11b) reflects a decrease in the motional characteristic frequency. Therefore larger amounts of pheromone produce a reduction in the mobility of water molecules. This is in good agreement with the reduction of the inter-lamellar layers with increasing pheromone content observed by SAXS (see Table 4).

On the other hand, the measurement of ${ }^{2} \mathrm{H} T_{1}$ for the DOPC/ $\mathrm{H}_{2} \mathrm{O} /$ cyclohexane- $\mathrm{d}_{12}$ samples provides some information on the dynamics of the host molecule and the aliphatic chains of the phospholipid. Fig. 11c shows that the relaxation time increases with the amount of uptaken cyclohexane, indicating an increased characteristic frequency of the motion contributing to $T_{1}$ that should correspond to an increased mobility of the aliphatic phospholipid chains, in agreement with the results obtained from the ${ }^{13} \mathrm{C}$ spectra.

\section{Conclusions}

The uptake of a very strong hydrophobic guest (1,7-dioxaspiro[5.5] undecane or "olean") by a DOPC/water lamellar phase was investigated through SAXS, DSC and NMR. The results of this multidisciplinary investigation indicate that the addition of the hydrophobe has a significant impact on the phase structure of the lipid bilayer, bringing about an $\mathrm{L}_{\alpha}-\mathrm{H}_{\mathrm{II}}$ phase transition followed by an $\mathrm{H}_{\text {II }}-\mathrm{I}_{\text {II }}$ phase change. The NMR results confirm the hydrophobe in the phospholipid chains domains, and in particular in proximity to the terminal methyl groups. The phase changes induced by the uptake of the hydrophobe also slow down the water reorientational motions and lower its freezing temperature, both related to increasing water confinement.

SAXS data confirm the structural changes detected by NMR experiments. DSC scans indicate the $\mathrm{L}_{\mathrm{c}}-\mathrm{L}_{\alpha}$ phase transition and the water melting change, depending on the uptake of olean into the DOPC/water nanostructure.

In conclusion, these results seem to confirm that the penetration of a strong hydrophobe (in this case a pheromone) in a biomimetic lipid bilayer (DOPC/water) has profound consequences on the packing of the lipid molecules in the self-assembly, and also on the distribution and properties of the hydration water molecules. Actually the packing change in the lipid and the properties of the aqueous pool seem to be two intertwined phenomena that mutually affect one another. More work is needed to deepen the understanding of this important issue.

These findings point out that the interplay of phase changes and variation in the hydration of the lipid polar head groups can be - at least partly - involved in the mechanism that drives the nerve conduction process in all the natural and physiological cases in which it is at work.

\section{Acknowledgements}

The authors gratefully acknowledge CSGI for partial financial support and the Enzo Ferroni Foundation for helpful discussions.

\section{References}

1 B. W. Ninham and P. Lo Nostro, Molecular Forces and Self Assembly. In Colloid, Nano Sciences and Biology, Cambridge University Press, Cambridge, UK, 2010. 
2 S. Turkyilmaz, P. F. Almeida and S. L. Regen, Langmuir, 2011, 27, 14380.

3 R. Reigada, J. Phys. Chem. B, 2011, 115, 2527.

4 Z. Yi, M. Nagao and D. P. Bossev, J. Phys.: Conf. Ser., 2010, 251, 012037.

5 H. Tsuchiya, T. Ueno, M. Mizogami and K. Takakura, Chem.-Biol. Interact., 2010, 183, 19.

6 T. T. Nguyen, J. L. Swift, M. C. Burger and D. T. Cramb, J. Phys. Chem. B, 2009, 113, 10357.

7 D. Huang, T. Zhao, W. Xu, T. Yang and P. S. Cremer, Anal. Chem., 2013, 85, 10240.

8 K. Hichiri, O. Shirai and K. Kano, Anal. Sci., 2012, 28, 45.

9 N. P. Franks, Nat. Rev. Neurosci., 2008, 9, 370-386.

10 J. A. Campagna, K. W. Miller and S. A. Forman, N. Engl. J. Med., 2003, 348, 2110.

11 A. Kopp Lugli, C. S. Yost and C. H. Kindler, Eur. J. Anaesthesiol., 2009, 26, 807.

12 E. N. Brown, R. Lydic and N. D. Schiff, N. Engl. J. Med., 2010, 363, 2638.

13 I. Ueda and T. Yoshida, Chem. Phys. Lipids, 1999, 101, 65.

14 I. Ueda, H. S. Tseng, Y. Kaminoh, S.-M. Ma, H. Kamaya and S. H. Lin, Mol. Pharmacol., 1986, 29, 582.

15 I. Ueda, Keio J. Med., 2001, 50, 20.

16 M. Baciu, M. C. Holmes and M. S. Leaver, J. Phys. Chem. B, 2007, 111, 909.

17 B. W. Urban, M. Bleckwenn and M. Barann, Pharmacol. Ther., 2006, 111, 729.

18 R. Søgaard, T. M. Werge, C. Bertelsen, C. Lundbye, K. L. Madsen, C. H. Nielsen and J. A. Lundbaek, Biochemistry, 2006, 45, 13118.

19 H. Tsuchiya, T. Ueno, M. Mizogami and K. Takakura, Chem.-Biol. Interact., 2010, 183, 19.

20 X. Wang and Q.-X. Jiang, J. Med. Mol. Biol., 2012, 9, 425.

21 M. Weinrich and D. L. Worcester, J. Phys. Chem. B, 2013, 117, 16141.

22 K. Larsson, Langmuir, 1988, 4, 215.

23 C. Bandeiras, A. P. Serro, K. Luzyanin, A. Fernandes and B. Saramago, Eur. J. Pharm. Sci., 2013, 48, 153.

24 M. Weinrich, H. Nanda, D. L. Worcester, C. F. Majkrzak, B. B. Maranville and S. M. Bezrukov, Langmuir, 2012, 28, 4723.

25 H. Tsuchiya, T. Ueno, M. Mizogami and K. Takakura, J. Anesth., 2010, 24, 639.

26 M. Engelke, R. Jessel, A. Wiechmann and H. A. Diehl, Biophys. Chem., 1997, 67, 127.

27 D. Uhríková, G. Rapp, S. Yaradaikin, V. Gordeliy and P. Balgavý, Biophys. Chem., 2004, 109, 361.

28 S. Turkyilmaz, W.-H. Chen, H. Mitomo and S. L. Regen, J. Am. Chem. Soc., 2009, 131, 5068.

29 M. A. Knackstedt and B. W. Ninham, Phys. Rev. E: Stat. Phys., Plasmas, Fluids, Relat. Interdiscip. Top., 1994, 50, 2839.

30 K.-E. Kaissling, Chem. Senses, 1996, 21, 257.

31 K. Mori, Chem. Commun., 1997, 1153.

32 K. Takeda, Y. Sano, S. Ichikawa, Y. Hirata, H. Matsuki and S. Kaneshina, J. Oleo Sci., 2009, 58, 369.

33 L. Stowers and T.-H. Kuo, Curr. Opin. Neurobiol., 2015, 34, 103. 34 J. Y. Yew and H. Chung, Prog. Lipid Res., 2015, 59, 88.
35 R. M. McKinney, C. Vernier and Y. Ben-Shahar, Curr. Opin. Insect Sci., 2015, 12, 86.

36 C. A. Oi, J. S. van Zweden, R. C. Oliveira, A. van Oystaeyen, F. S. Nascimento and T. Wenseleers, BioEssays, 2015, 37, 808.

37 J. Nardella, M. Terrado, N. S. Honson and E. Plettner, Arch. Biochem. Biophys., 2015, 579, 73.

38 A. Papachristoforou, A. Kagiava, C. Papaefthimiou, A. Termentzi, N. Fokialakis, A.-L. Skaltsounis, M. Watkins, G. Arnold and G. Theophilidis, PLoS One, 2012, 7, e47432.

39 A. S. Ulrich, M. Sami and A. Watts, Biochim. Biophys. Acta, 1994, 1191, 225.

40 D. G. Cory and W. M. Ritchey, J. Magn. Reson., 1988, 80, 128.

41 K. Eichele and R. E. Wasylishen, WSOLIDS NMR Simulation Package, 2000.

42 T. Blanton, T. C. Huang, H. Toraya, C. R. Hubbard, S. B. Robie, D. Louer, H. E. Gobel, G. Will, R. Gilles and T. Raftery, Powder Diffr., 1995, 10, 91.

43 J. A. Lake, Acta Crystallogr., 1967, 23, 191.

44 R. P. Rand and N. L. Fuller, Biophys. J., 1994, 66, 2127.

45 S. C. Costigan, P. J. Booth and R. H. Templer, Biochim. Biophys. Acta, 2000, 1468, 41.

46 S. Tristram-Nagle, H. I. Petrache and J. F. Nagle, Biophys. J., 1998, 75, 917.

47 M. Sjölund, G. Lindblom, L. Rilfors and G. Arvidson, Biophys. J., 1987, 52, 145.

48 R. N. A. H. Lewis, B. D. Sykes and R. N. McElhaney, Biochemistry, 1988, 27, 880.

49 T. Heimburg and A. D. Jackson, PNAS, 2005, 102, 9790.

50 E. Rowe, Mol. Pharmacol., 1982, 22, 133.

51 R. Winter and C. Jeworrek, Soft Matter, 2009, 5, 3157.

52 A. S. Ulrich and A. Watts, Biophys. Chem., 1994, 49, 39.

53 A. Nilsson, A. Holmgren and G. Lindblom, Biochemistry, 1991, 30, 2126.

54 C. Dolle, P. Magrone, S. Riva, M. Ambrosi, E. Fratini, N. Peruzzi and P. Lo Nostro, J. Phys. Chem. B, 2011, 115, 11638.

55 Z. Chen, L. C. M. Van Gorkom, R. M. Epand and R. E. Stark, Biophys. J., 1996, 70, 1412.

56 C. P. S. Tilcock, P. R. Cullis and S. M. Gruner, Chem. Phys. Lipids, 1986, 40, 47.

57 J. Mason, Solid State Nucl. Magn. Reson., 1993, 2, 285.

58 F. Volke, S. Eisenblatter, J. Galle and G. Klose, Chem. Phys. Lipids, 1994, 70, 121.

59 Z. Zhou, B. G. Sayer, D. W. Hughes, R. E. Stark and R. M. Epand, Biophys. J., 1999, 76, 387.

60 G. Chidichimo, N. A. P. Vaz, Z. Yaniv and J. W. Doane, Phys. Rev. Lett., 1982, 49, 1950.

61 P. L. Hubbard, K. M. McGrath and P. T. Callaghan, J. Phys. Chem. B, 2006, 110, 20781.

62 L. M. Strenk, P. W. Westerman, N. A. P. Vaz and J. W. Doane, Biophys. J., 1985, 48, 355.

63 R. Poupko and Z. Luz, J. Chem. Phys., 1981, 75, 1675.

64 N. Boden, S. A. Jones and F. Sixl, J. Am. Chem. Soc., 1991, 30, 2146.

65 B. Cowan, Nuclear Magnetic Resonance and Relaxation, Cambridge University Press, Cambridge, UK, 1997. 